\title{
Structure Identification and Quality Assessment of Laccase (Lac InaCC) from Neurospora crassa by Using a Structure Prediction
}

\author{
Rini Kurniasih", Laksmi Ambarsari ${ }^{1 *}$, Setyanto Tri Wahyudi \\ ${ }^{1}$ Department of Biochemistry, Bogor Agricultural University, Bogor, Indonesia \\ ${ }^{2}$ Department of Physics, Bogor Agricultural University, Bogor, Indonesia
}

\section{ARTICLE INFO}

Article history:

Received October 19, 2019

Received in revised form November 15, 2020

Accepted November 20, 2020

\section{KEYWORDS:}

laccase,

Neurospora crassa,

sequence motifs,

structure prediction,

molecular dynamics simulation

\begin{abstract}
Laccases are multi-copper oxidase enzyme, developed for being applied widely. The laccase gene in this study was isolated from local isolates of Neurospora crassa (LAC inaCC). The structure of this enzyme has not been known and there is no laccase structure of Neurospora crassa based on protein structure development in database. Here, we aimed to analyze the characteristics of the sequence and prediction structure, the structure quality after refinement through the molecular dynamics (MD) simulation method. LAC inaCC has been identified with typical sequence motifs (HWH, HSH, HXXH) which played role in copperbinding on 274(HWH)G-DG-T-CP on CBL-1, 314GT-WY(HSH)FS-QYG-G on CBL-2, and 607HPIHL on CBL-3. The four copper atoms have an important role in the catalytic activity. $\mathrm{LAC}$ inaCC is a multi-subunit enzyme consisted of three functional domains with structural motifs of Greek-key $\beta$ barrel which is typical structure motif. Refinement in the prediction structure through the MD simulation showed that this method was proven to be able to improve the structure quality. The increase on the most favoured area on Ramachandran plot, clashcore percentile score, and molprobity score showed that the laccase structure headed to conformation change, to be more stable conformation with better resolution compared to earlier prediction structure.
\end{abstract}

\section{Introduction}

Laccases are oxidoreductase enzymes with EC 1.10.3.2, the enzyme that catalyzes substrate with polyphenol aromatic substance as the donor of electron and involves oxygen as the recipient of the electron to form water molecule. Laccases are enzymes found in bacteria, fungi, and plants. Laccases play different roles in each organism. In fungi, laccases act differently with ones in plants, where laccases play a role in lignin degradation contained in the cell wall of the host plants (Dwivedi et al. 2011). The differences in laccase roles in each organism caused laccases catalytic properties vary depending on the sources, such as various types of thermostability and substrate. Catalytic and functional properties of the enzyme are closely related to characteristics at the molecular level, i.e., the genetic sequence and the structure of the enzyme. Understanding the properties and functions of catalytic enzymes at the molecular level in the computational simulation is faster, more

* Corresponding Author

E-mail Address: laksmi@apps.ipb.ac.id efficient, and more cost-effective than the laboratory experiment.

Laccase analyzed in this study was from our previous research, where the laccase gene was isolated from Neurospora crassa code of inaCC F226 fungi. The structure of LAC inaCC has not been known and there is no laccase structure of Neurospora crassa fungi based on protein structure development in the laccase database of Biocatnet LccED V6.4 yet. The purposes of this research were to predict the structure of LAC inaCC, to identify the sequence characteristics, to identify the laccase prediction structure, and to ameliorate the prediction structure conformation obtained. The structure of LAC inaCC in this research was obtained from structure prediction by using the fold recognition method, I-TASSER.

Protein structure quality appraisal plays an important role in the structure prediction of protein, as the structure prediction of protein might contain a substantial error (Benkert et al. 2011). Refinement on such structures is necessary if the prediction has poor results (Benkert et al. 2011). The refinement of the structure prediction of LAC inaCC in this research used the molecular dynamics (MD) simulation method. 
The MD simulation method is known to be able to ameliorate the structure prediction of protein (Feig 2016; Feig and Mirjalili 2016).

\section{Materials and Methods}

\subsection{Materials}

The nucleotide sequence of laccase, the amino acid sequence of laccase from the LAC inaCC gene was translated by using the sequence translation sites, 17 amino acid sequences and PDB files consisting of laccase and the multicopper oxidase (MCO) enzymes from homologous sequences of LAC inaCC based on BLASTp homology analysis and the template of the modeling structure of LAC inaCC. The laccase's structure was predicted by I-TASSER.

\subsection{Instruments}

The high-performance computer (HPC) with Linux Ubuntu system 64 bit, identification of sequence and structure characteristics using the sequence translation site from the European Bioinformatics Institute (EBI), homology analysis BLASTp, the conserved domain architecture retrieval tool (CDART), and the multiple sequences alignment by Kalign. Assessment of protein structure quality before refinement was carried out using PDBsum and molprobity. Molecular dynamics simulation with Assisted model building with energy refinement (AMBER) system version 12 for assessment and refinement of the prediction structure of LAC inaCC. The Protein structure visualization was carried out using Pymol dan Visual Molecular Dynamics (VMD) version 1.9.2.

\subsection{Homologous Analysis}

The nucleotide sequence of LAC inaCC was translated into the amino acid sequence via the Emboss_Transeq sequence translation server from the European Bioinformatics Institute (EBI). Then, the homology of the amino acid sequence was analyzed using BLASTp (https://blast.ncbi.nlm.nih.gov/Blast. cgi), to determine the similarity of the amino acid sequences to the amino acid sequences in the Protein Data Bank (PDB) database.

\subsection{Amino Acids Sequences Characterization}

The amino acid sequence was characterized to prove that it is a laccase gene. Characteristics analyzed were the sequence motif and the copper-binding site of laccase by using the multiple sequence alignment method, Kalign server. That sequence aligned with 17 amino acid sequence of laccase and other enzymes from the multicopper oxidase (MCO) superfamily. The determination of sequence motif and copper-binding site of LAC inaCC referred to Giardina et al. (2009).
Then, some sequences that play a role in the sequence motif and copper-binding site were visualized into sequence logos by using a web logo (https://weblogo. berkeley.edu/logo.cgi).

\subsection{LAC inaCC Structure Prediction}

The amino acid sequence resulted from the Emboss Transeq EBI was used to predict the structure of LAC inaCC. The structure of LAC inaCC was predicted by using the fold recognition method, I-TASSER. The amino acid sequence was submitted to I-TASSER, http://zhanglab. ccmb.med.umich.edu/I-TASSER/. The prediction is started by clicking the "RUN I-TASSER" icon. Then, the best prediction structure of LAC inaCC resulted in I-TASSER was chosen, model 1 was chosen as the best predictive structure. The quality of the prediction structure produced by I-TASSER was evaluated for the first time using the web server protein structure quality, including 1) PDBsum, http://www.ebi.ac.uk/ pdbsum/, to see structural conformational stability through Ramachandran plots and percentage regions of most favored region and disallowed region, 2) proQ https://proq.bioinfo.se/ProQ/ProQ.html, to predict protein structure quality by LGscore and MaxSub, 3) molprobity, http://molprobity.biochem.duke.edu/, to evaluate the model quality of protein at both the global and local level by molprobity score.

\subsection{Structure Characterization}

The structure prediction of LAC inaCC was conducted to analyze its characteristics such as domains, structural motifs, active sites, and copperbinding sites. The domain was analyzed using CDART, structural motifs were analyzed using Pymol and VMD, active site and copper-binding site were analyzed using the multiple sequence alignment method (Kalign) and PDBsum, then they were visualized by Pymol.

\subsection{Refinement of The Prediction Structure of LAC inaCC by MD Simulation}

The structure prediction of LAC inaCC was refined by molecular dynamics simulation method. This simulation was conducted using AMBER software, with the ff14SB force field that was recommended for protein. Lacase is a metalloprotein consisting of three copper atoms. Parameterization (preparation stage) of three copper atoms is carried out using the MCPB. py Version 2.0 method which has been released in AmberTools16. The explicit solvent model was chosen for laccase's structure simulation with water solvent (code: TIP3PBOX) without adding $\mathrm{Cl}^{-}$and $\mathrm{Na}^{+}$. First, the structure was prepared in a box with a distance between protein and the box's wall was $20 \AA$. The structure was subsequently minimized using an all-atom force field 
on $\mathrm{C} \alpha$ atoms with respect to the initial model. Applying constraints in minimization was necessary because the initial model may be energetically very unfavorable and could cause the conformation alteration due to high initial strain energies (Shen et al. 2015; Feig 2016). Then, the equilibration and heating step was conducted using NPT condition with temperature in an interval of $0 \mathrm{~K}$ to $310 \mathrm{~K}$. On the final step, the structure simulation is conducted in NPT condition with a temperature of $310 \mathrm{~K}$ for $100 \mathrm{~ns}$ time simulation.

\subsection{Assessment of The Prediction Structure of LAC inaCC Before and After Refinement by MD Simulation}

Assessment of the structure quality of LAC inaCC was conducted using PDBsum to obtain the Ramachandran plot with the percentage of amino acid distribution regions, and molprobity to obtain claschore and molprobity score for describing the resolution of the structure prediction. Meanwhile, the structure prediction of laccase after refinement by MD simulation was analyzed by using PDBsum, molprobity, and some parameters of molecular dynamics such as root mean square deviation (RMSD), root mean square fluctuation (RMSF), interaction energy, and the solvent-accessible surface area (SASA).

\section{Results}

The laccase (LAC inaCC) gene had the molecular weight of $1,900 \mathrm{bp}$ with a similarity percentage of 95-97\% towards laccase from a different strain of Neurospora crassa and Neurospora tetrasperma. The homology of the amino acid sequence from translation was not high. The highest similarity (Table 1 ) was ones to laccase of Melanocorpus albomyces and Thielavia arenaria with similarity percentage (identic) of
$63 \%$, this was because Neurospora crassa is a fungus from the same ordo with Melanocorpus albomyces and Thielavia arenaria (Phylum of Ascomycetes and ordo of Sordariales). Meanwhile, the similarity with laccase sequences from other organisms was only about 34-35\%. Through multiple sequence alignment it is possible to identify enzyme groups by analyzing sequence motifs especially for sequences that have a low level of similarity such as LAC InaCC which only has the highest similarity of $63 \%$.

This enzyme belongs to the multicopper oxidase (MCO) superfamily of enzyme and has three sequence motifs of $\mathrm{H}-\mathrm{X}-\mathrm{H}$ as shown in Figure 1. The sequence motifs of $\mathrm{H}-\mathrm{X}-\mathrm{H}$ consisting of $\mathrm{HWH}, \mathrm{HSH}$, and $\mathrm{HXXH}$. The HWH motif is located on the tip of $\mathrm{N}$ area. Meanwhile, the HSH and HXXH are on the tip of Carea. These three motifs $\mathrm{H}-\mathrm{X}-\mathrm{H}$ are part of amino acid residue interacting with copper atoms (CU). All of the sequence motifs of laccases were designed to be sequence logo which can be seen in Figure 2. The sequence motif is not only used to identify a group of the enzyme but also can be used to identify the functional site of the enzyme, such as catalytic site, binding site, structural motif, etc. The three $\mathrm{H}-\mathrm{X}-\mathrm{H}$ motifs are related to copper atom binding site involving the most conserved histidine site.

The determination of copper atom binding site was known by laccase amino acid sequence alignment. There were four loops of copper-binding sites involving the $\mathrm{H}-\mathrm{X}-\mathrm{H}$ motifs and a number of amino acids. There were three loops of copper-binding site found in LAC inaCC, named copper-binding loop (CBL), with a number of conserved amino acid residues in each loop, in Table 2 and Figure 3. The conserved amino acid residues in each copper-binding loop were ${ }^{274}(\mathrm{HWH})$ G---DG---T-CP on CBL-1, ${ }^{314}$ GT-WY(HSH)FS-QYG-G--on CBL-2, meanwhile the CBL-3 consisted of only five amino acids, they were ${ }^{607} \mathrm{HPIHL}$. These conserved

Table 1. Homology parameters of the amino acid sequence of LAC inaCC

\begin{tabular}{|c|c|c|c|c|c|c|}
\hline Organism & Max score & Total score & Query cover \% & E value & Ident \% & Accesion \\
\hline $\begin{array}{l}\text { Laccase Melanocarpus albomyces mutant } \\
\text { L559A }\end{array}$ & 599 & 599 & 72 & 0.0 & 63 & 3DKH_A \\
\hline Laccase Melanocarpus albomyces & 599 & 599 & 72 & 0.0 & 63 & 1GW0_A \\
\hline $\begin{array}{l}\text { Laccase Melanocarpus albomyces Near- } \\
\text { atomic resolution structure }\end{array}$ & 596 & 596 & 72 & 0.0 & 63 & 2Q90_A \\
\hline Laccase Thielavia arenaria & 585 & 585 & 73 & 0.0 & 63 & 3PPS_A \\
\hline Laccase Botrytis aclada mutant L499M & 274 & 274 & 81 & $7 e-84$ & 35 & 3V9E־_A \\
\hline Laccase Botrytis aclada at $1.67 \AA ̊$ resolution & 274 & 274 & 81 & $7 e-84$ & 35 & 3SQR_A \\
\hline $\begin{array}{l}\text { Multicopper oxidase Aspergillus niger } \\
\text { mutant H253D }\end{array}$ & 244 & 244 & 70 & $2 \mathrm{e}-72$ & 34 & 5LWX_A \\
\hline $\begin{array}{l}\text { Laccase-like-Multicopper oxidase } \\
\text { Aspergillus niger bound to zinc }\end{array}$ & 243 & 243 & 70 & $2 \mathrm{e}-72$ & 34 & 5LWW_A \\
\hline $\begin{array}{l}\text { Laccase-like-Multicopper oxidase } \\
\text { Aspergillus niger }\end{array}$ & 243 & 243 & 70 & $2 \mathrm{e}-72$ & 34 & 5LM8_A \\
\hline Laccase Lentinus sp. At $1.8 \AA \AA$ resolution & 179 & 179 & 62 & $3 e-49$ & 34 & 3X1B_A \\
\hline
\end{tabular}


N. crassa(LaccI16) 270 GTS HWH GMHORNSNIQDGVNGVTECPIPP 299 Zhucini(1AOZ)* 5 GWVHHWGILORGTPWADGTASISOCAINP 85 B.aclada(3SOR) 120 GTSIHWIGIROLGSLEYDGVPGVTOCPIAP 149 B.aclada(3V9E) $12 a \mathrm{GTS}$ HHWIGIRQLGSLEYDGVPGVTQCPIAP 16 C.galicca(4A2H) GSTSHWHGFFOHGTMWADGPAFVNOCPIST Lentinus. $5 p$ (3X1B) 81 STS HWW GGFFOHGTMWADGGAFVNOCPISS 110 M.albomyces (3FU7) "9GS IHWHGIHOKDTNLHDGANGVTECPIPP 118 M.albomyces (1GMO) GTS HHWGGIHOKDTNLHDGANGVTECPIPP 118 M.albonyces (2Q90) ag GTS HWH GIHOKDTNLHDGANGVTECPIPP 118 M.albanyces (3DKH) ${ }^{89}$ GTS HHWIGIHOKDTNLHDGANGVTECPIPP 118 A.niger (5LWW * nGTA_HFHGIRQLYNNOMDGVAALTQCPVPP 106 A.niger (5LM8)* 76 GTA_HFHGIRQLYNNOMDGVAALTQCPVPP 105 A.niger $(5 L \mathrm{LX})^{*}$ La4 GTAIHFHGIRQLYNNOMDGVAALTQCPVPP 133 S. ochraceum (3T6V) 61 DTS.HWH GEEFOKGTNWADGPAFITQCPIIV 90 T.versicolor(1GYC) w STS HWH GGFFOAGTNWADGPAFVNOCPIAS 89 T. versicolor(1KYA) 60 STS IHWHGFFOKGTMWADGPAFINOCPISS 89 T. hirsuta(3FPX) GS STS HWWIGFFOHGTMWADGPAFINOCPISP Th.arenaria(3PPS) 130 GTSI) HWHGLLRQLGNVFNDGANGVTECPIPP 159
N. crassa(LaccI16) 311 TQYGTSW HSHFSAQYGNGIVGPIVI 213 Zhucini(1AOZ)* $\%$ DNPGTFF HGG GMORSAGLYGSLIV 135 B.aclada(3SOR) 160 TQYGTTMHSIFESLOYGDGLFGPLII 185 B.aclada(3V9E) 160 TQYGTTMHSSF FSLQYGDGLFGPLII ${ }^{165}$ C.galicca(4A2H) in DQAGTFMHSH STOYCDGLRGPIW 103 Lentinus.SP(3X1B) 12 DQAGTFMHSH STOYCDGLRGPFW 17 M.albonyces(3FU7) 130 RQYGTSMHSSHFSAQYGNGWGTIQI ${ }^{167}$ M.albanyces(1GM) 130 RQYGTSWHSSHFSAQYGNGWGTIOI Iss M.albanyces (2090) 130 RQYGTSMHHSHFSAQYGNGWGTIQI 155 M.albonyces(3DKH) 130 RQYGTSWHSIF FSAQYGNGWGTIQI 155 A.niger (5LWW* ${ }^{*}$ EEYGSSWHSSHESLQANEGVFGGILI 142 A.niger (5LM8)* 116 EEYGSSWHHSIFSLLANEGVFGGILI 14 A.niger $(5 L \mathrm{LWX})^{*}$ 14 EEYGSSWMHSHFSLQANEGVFGGILI 169 S.ochraceum(3T6V) 102 GMAGTM/HSH LTTQYCDGLRGPFW 14 T.versicolor(1GYC) an DQAGTFMHSH STOYCDGLRGPFW 163 T.versicolor(1KYA)101 DQAGTFMHSH.SSTQYCDGLRGPFW 103 T.hirsuta(3FPX) 101 DQAGTFMHSH STOYCDGLRGPFW 14 Th.arenaria(3PPS) 17 TQYGTSWHSSH FSAQYGNGWGTIQI 198

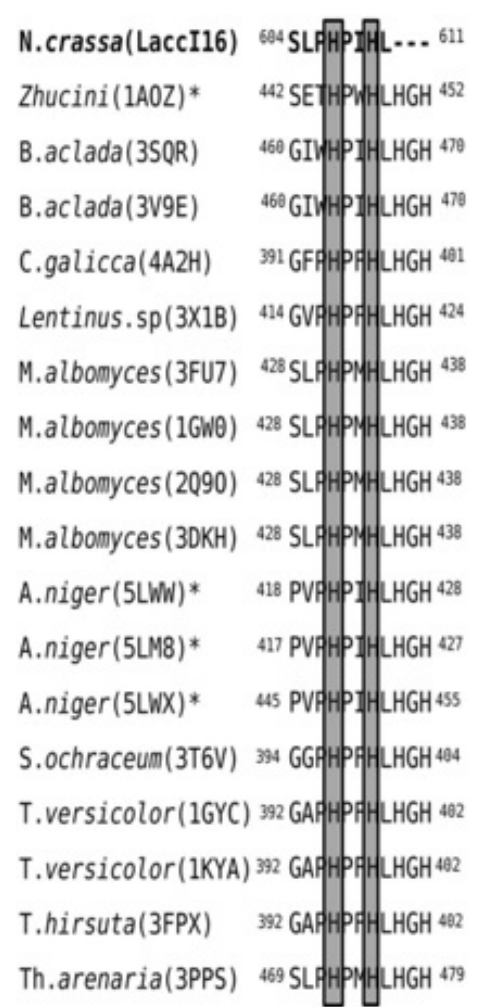

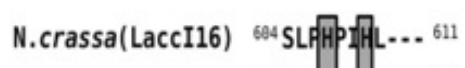
Zhucini(1AOZ)*

aclada(3SOR)

B.aclada(3V9E)

.gaticca $(4 \mathrm{~A} 2 \mathrm{H})$

Lentinus.sp ( $3 \times 1 \mathrm{~B})$

(a)

438

M.albomyces (1GWO) 428 SLPHPPH LLHGH ${ }^{438}$

M.albomyces (2090) 428 SLPHPP HL LHGH 438

M.albomyces (3DKH) ${ }^{428}$ SLPH PP HL LHGH ${ }^{438}$

A.niger (5LWW)*

418 PVAHPI ILLHGH 428

PI

A.niger (5LWX)

455

\section{2}

T.versicolor(1KYA) 392 GAA IPPH LHGH 402

Th. arenaria(3PPS) ${ }^{469}$ SLPH HPH HLHGH 479

Figure 1. Amino acid sequence alignment of LAC inaCC with laccases and other enzymes from the different organisms by Kalign. The other enzymes are shown with asterisks, they are the multicopper oxidase from Aspergillus niger. The LAC inaCC sequence is shown in bold. The black box is the laccase sequence motif (the conserved sequence)

a

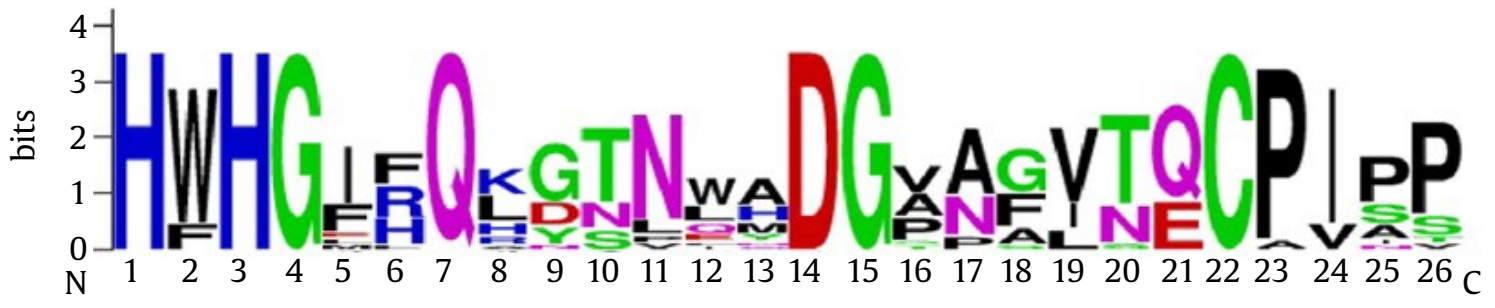

b
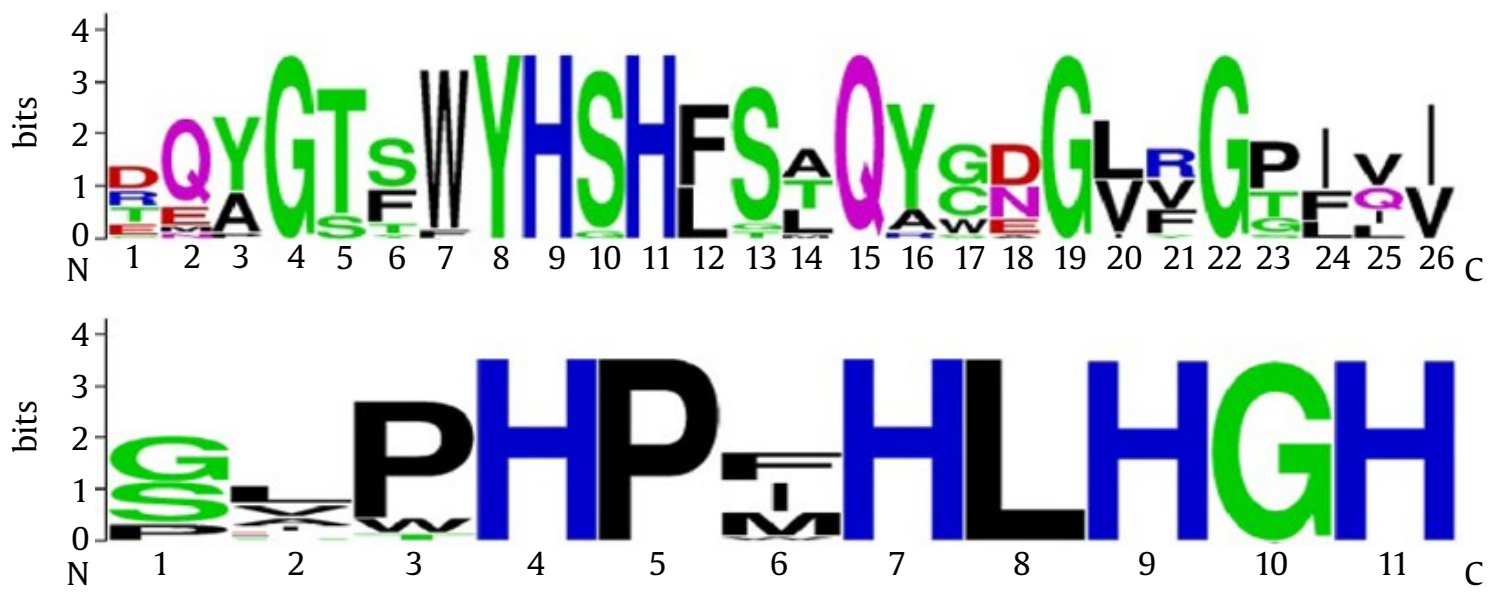

Figure 2. Logo sequences for the copper-binding residues of LAC inaCC. The first, second and third of copper-binding residues are shown in the a, b and c logo sequences. The sequence logos were created by using web logo 
amino acids were expected to have an important role in the catalytic activity of laccase.

The structure prediction of LAC inaCC by using I-TASSER, showed that the enzyme is a multisubunit monomer enzyme consisting of three functional subunits known as cupredoxin-like domain. Three domains composing this enzyme are domain $\mathrm{A}$ (1341), domain B (342-500), and domain C (501-611), in Figure 4 . This enzyme contained four copper atoms, showed in Figure 4a. The four copper atoms showed that the enzyme belongs to a group of multicopper oxidase (MCO) enzyme.

In addition to domain analysis and number of copper atoms, several other characteristics were also analyzed to identify that the LAC inaCC structure predicted by iTASSER is a lacase structure such as the substrate pocket and the substrate-binding loop and the substrate pocket. The analysis of substratebinding loop result showed that the LAC inaCC had four substrate-binding loops, they were loop I to loop IV and can be seen in Figure 5a. The conserved amino acid residues in the substrate-binding loops of the protein were $(X) A D(X) T S(X) N H F$ on loop I, $\operatorname{VTFG}(\mathrm{X}) \mathrm{CG}(\mathrm{X})$ on loop II, $\mathrm{LD}(\mathrm{X}) \mathrm{FVW}$ on loop III, and the residue of YWLIE(X)PHP(X)H(X) on loop IV.

The substrate pocket of LAC inaCC was composed of a number of amino acids located near to $\mathrm{T} 1$ copper (the site between domain A and B). The T1 copper and the site around it are the location of substrate oxidation of laccase (active site) in Figure 5. Most of the substrate pocket of this enzyme was composed of hydrophobic residues, they are Phe322, Phe351, Ala361, Val365, Leu366, Ala555, Ile556, Phe419, and Trp560, and the hydrophilic residues are Ser323, Asp362, Gln369, Asp545, and Asn557, showed at Figure $5 b$ and $c$.

The quality of the structure prediction of LAC inaCC was analyzed through PDBsum and molprobity. In the Ramachandran plot, the red zone is the most favoured regions, the brown zone is the additional allowed regions, the yellow zone is the generously allowed regions, and the white zone is the disallowed regions. Blue dot is shown amino acid residue. Based on Ramachandran plot from PROSCHEK on PDBsum, the structure prediction of

Table 2. The copper-binding loop of LAC inaCC

\begin{tabular}{|c|c|c|c|c|}
\hline Organisme & Id & $\begin{array}{l}\text { Copper- } \\
\text { binding } \\
\text { loop } 1\end{array}$ & $\begin{array}{l}\text { Copper- } \\
\text { binding } \\
\text { loop } 2\end{array}$ & $\begin{array}{l}\text { Copper- } \\
\text { binding } \\
\text { loop } 3\end{array}$ \\
\hline $\begin{array}{l}\text { Neurospora } \\
\text { crassa }\end{array}$ & - & ${ }^{274} \mathrm{H}^{\mathrm{a}}$ WHGMHQRNSNIQDGVNGVTECP & ${ }^{314}$ GTSWY HSH ${ }^{\mathrm{a}}$ FSAQYGNGIVGPI & ${ }^{607}$ HPI Ha L - - - \\
\hline Zuchini & $1 \mathrm{AOZ}$ & ${ }^{60} \mathrm{HWHG}$ ILQRGTPtiWADGTASISQCA & 999TFFYHGHLGMQRSAGLYGSL & ${ }^{445} \mathrm{HPWHLHGH}$ \\
\hline Botrytis aclada & 3SQR & ${ }^{124} \mathrm{HWHG}$ IRQLG SLEYDGVPGVTQCP & ${ }^{163}$ GTTWYHSHFSLQYGDGLFGPL & ${ }^{463} \mathrm{HP}$ I HLHGH \\
\hline Botrytis aclada & $3 \mathrm{~V} 9 \mathrm{E}$ & ${ }^{124} \mathrm{HWHG}$ IRQLG SLEYDGVPGVTQCP & ${ }^{163}$ GTTWYHSHFSLQYGDGLFGPL & ${ }^{463} \mathrm{HP}$ I HLHGH \\
\hline $\begin{array}{l}\text { Coriolopsis } \\
\text { galicca }\end{array}$ & $4 \mathrm{~A} 2 \mathrm{H}$ & ${ }^{64} \mathrm{HWHGFFQHGTNWADGPAFVNQCP}$ & ${ }^{104}$ GTFWYHSHLSTQYCDGLRGPI & ${ }^{394} \mathrm{HPFHLHGH}$ \\
\hline Lentinus.sp & 3X1B & ${ }^{85} \mathrm{HWHGFFQHGTNWADGGAFVNQCP}$ & ${ }^{125}$ GTFWYHSHLSTQYCDGLRGPF & ${ }^{417} \mathrm{HPFHLHGH}$ \\
\hline M. albomyces & 3FU7 & ${ }^{93} \mathrm{HWHGI}$ HQKDTNLHDGANGVTECP & ${ }^{133}$ GTSWYHSHFSAQYGNGVVGTI & ${ }^{431} \mathrm{HPMHLHGH}$ \\
\hline M. albomyces & $1 G W 0$ & ${ }^{93} \mathrm{HWHG}$ IHQKDTNLHDGANGVTECP & ${ }^{133}$ GTSWYHSHFSAQYGNGVVGTI & ${ }^{431} \mathrm{HPMHLHGH}$ \\
\hline M. albomyces & $2 Q 90$ & ${ }^{93} \mathrm{HWHG}$ IHQKDTNLHDGANGVTECP & ${ }^{133}$ GTSWYHSHFSAQYGNGVVGTI & ${ }^{431}$ HPMHLHGH \\
\hline M. albomyces & 3DKH & ${ }^{93} \mathrm{HWHG}$ IHQKDTNLHDGANGVTECP & ${ }^{133}$ GTSWYHSHFSAQYGNGVVGTI & ${ }^{431} \mathrm{HPMHLHGH}$ \\
\hline $\begin{array}{l}\text { Aspergillus } \\
\text { niger }\end{array}$ & 5LWW & ${ }^{81} \mathrm{HFHG}$ IRQLYNNQMDGVAALTQCP & ${ }^{120} \mathrm{GSSWYHSHFSLQAWEGVFGGI}$ & ${ }^{421} \mathrm{HP}$ I HLHGH \\
\hline $\begin{array}{l}\text { Aspergillus } \\
\text { niger }\end{array}$ & 5LM8 & ${ }^{80} \mathrm{HFHG}$ IRQLYNNQMDGVAALTQCP & ${ }^{119}$ GSSWYHSHFSLQAWEGVFGGI & ${ }^{420} \mathrm{HP}$ I HLHGH \\
\hline $\begin{array}{l}\text { Aspergillus } \\
\text { niger }\end{array}$ & 5LWX & ${ }^{108} \mathrm{HFHG}$ IRQLYNNQMDGVAALTQCP & ${ }^{117}$ GSSWYHSHFSLQAWEGVFGGI & ${ }^{448} \mathrm{HP}$ I HLHGH \\
\hline S.ochraceum & $3 \mathrm{~T} 6 \mathrm{~V}$ & 65 HWHGEFQKGTNWADGPAFITQCP & ${ }^{105}$ GTYWYHSHLTTQYCDGLRGPF & ${ }^{397} \mathrm{HPFHLHGH}$ \\
\hline $\begin{array}{l}\text { Trametes } \\
\text { versicolor }\end{array}$ & $1 G Y C$ & ${ }^{64} \mathrm{HWHGFFQAGTNWADGPAFVNQCP}$ & ${ }^{104}$ GTFWYHSHLSTQYCDGLRGPF & ${ }^{395}$ HPFHLHGH \\
\hline $\begin{array}{l}\text { Trametes } \\
\text { versicolor }\end{array}$ & 1KYA & ${ }^{64} \mathrm{HWHGFFQKGTNWADGPAFINQCP}$ & ${ }^{104}$ GTFWYHSHLSTQYCDGLRGPF & ${ }^{395}$ HPFHLHGH \\
\hline $\begin{array}{l}\text { Trametes } \\
\text { hirsuta }\end{array}$ & 3FPX & ${ }^{64} \mathrm{HWHGFFQHGTNWADGPAFINQCP}$ & ${ }^{104}$ GTFWYHSHLSTQYCDGLRGPF & ${ }^{395}$ HPFHLHGH \\
\hline $\begin{array}{l}\text { Thielavia } \\
\text { arenaria }\end{array}$ & 3PPS & ${ }^{134} \mathrm{HWHGLRQLGNVFNDGANGVTECP}$ & ${ }^{174}$ GTSWYHSHFSAQYGNGVVGTI & ${ }^{472}$ HPMHLHGH \\
\hline
\end{tabular}

a The copper-binding residue of LAC inaCC 


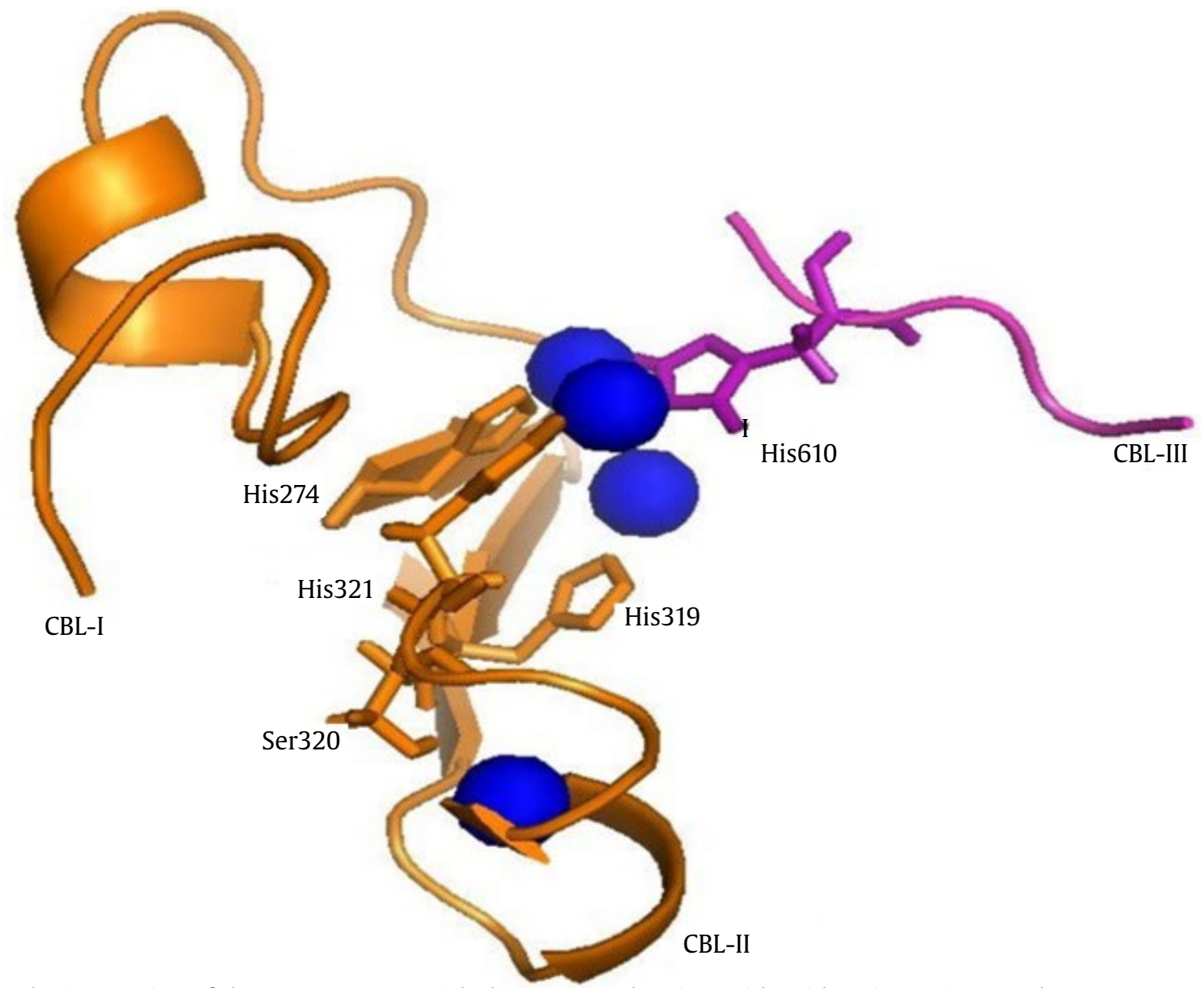

Figure 3. The interaction of the copper atoms with the conserved amino acid residues in LAC inaCC. The copper atoms are shown as a blue sphere, the dashed lines are hydrogen bond, and the colored sticks are the conserved amino acid residues
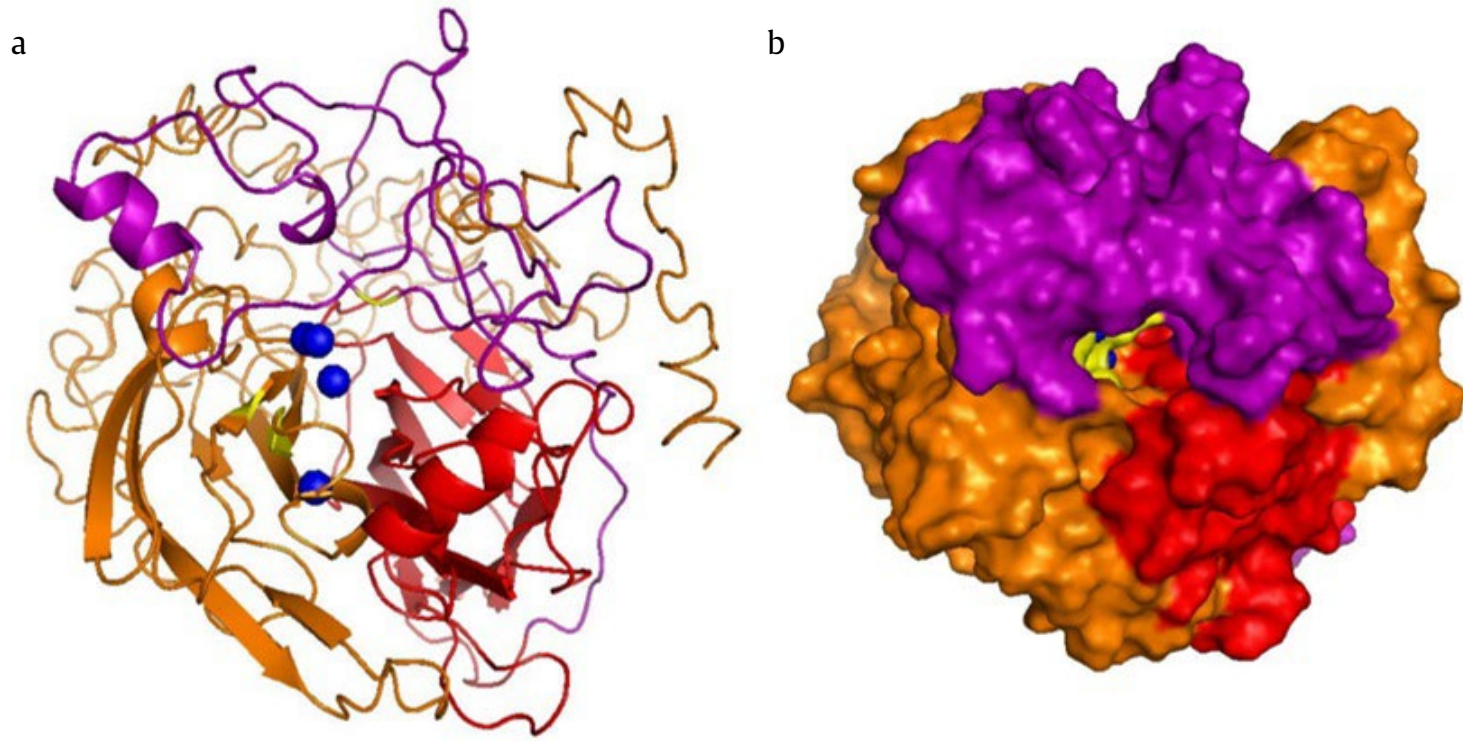

Figure 4. The structure prediction of LAC inaCC by using I-TASSER. (a) The structure prediction of the enzyme in new cartoon form, (b) the structure prediction of the enzyme in surface form. Domain A is colored by red, domain B is colored by orange, and domain $\mathrm{C}$ is colored by purple. The copper atoms are shown as a blue sphere 
LAC inaCC had a low most favored region percentage of $55.6 \%$ with pretty-high disallowed regions percentage of $2.8 \%$ (in Table 3), described in Figure 6a. The percentage of the most favored region increased significantly into 78.7\% after refinement through the MD simulation method, it was followed by an increase in the value of claschore and molprobity ( $99^{\text {th }}$ percentile and $89^{\text {th }}$ percentile).

Other parameters that indicate improvement in the quality of the structure after refinement through the MD simulation method are root mean square deviation (RMSD), root mean square fluctuation (RMSF), interaction energy, and the solvent- accessible surface area (SASA). Based on the RMSD graphic value towards the simulation time in Figure 7a, there was significantly increasing and fluctuation of RMSD value towards the simulation for $50 \mathrm{~ns}$. The continuous increasing of RMSD value showed a continuous alteration of the structure. The RMSD value was inclined to be stable at the simulation of $80 \mathrm{~ns}$ until the end of the simulation (100 ns) with the RMSD value of 3.0 A. It showed an alteration in structure conformation of LAC inaCC during the simulation towards the conformation stability. The fluctuation of amino acid positional alteration can be explained by using the SASA value. The SASA value showed the area of protein structure surface interaction towards solvents. The highest SASA value was obtained by the back-bone structure of protein as much as 4073 A22.

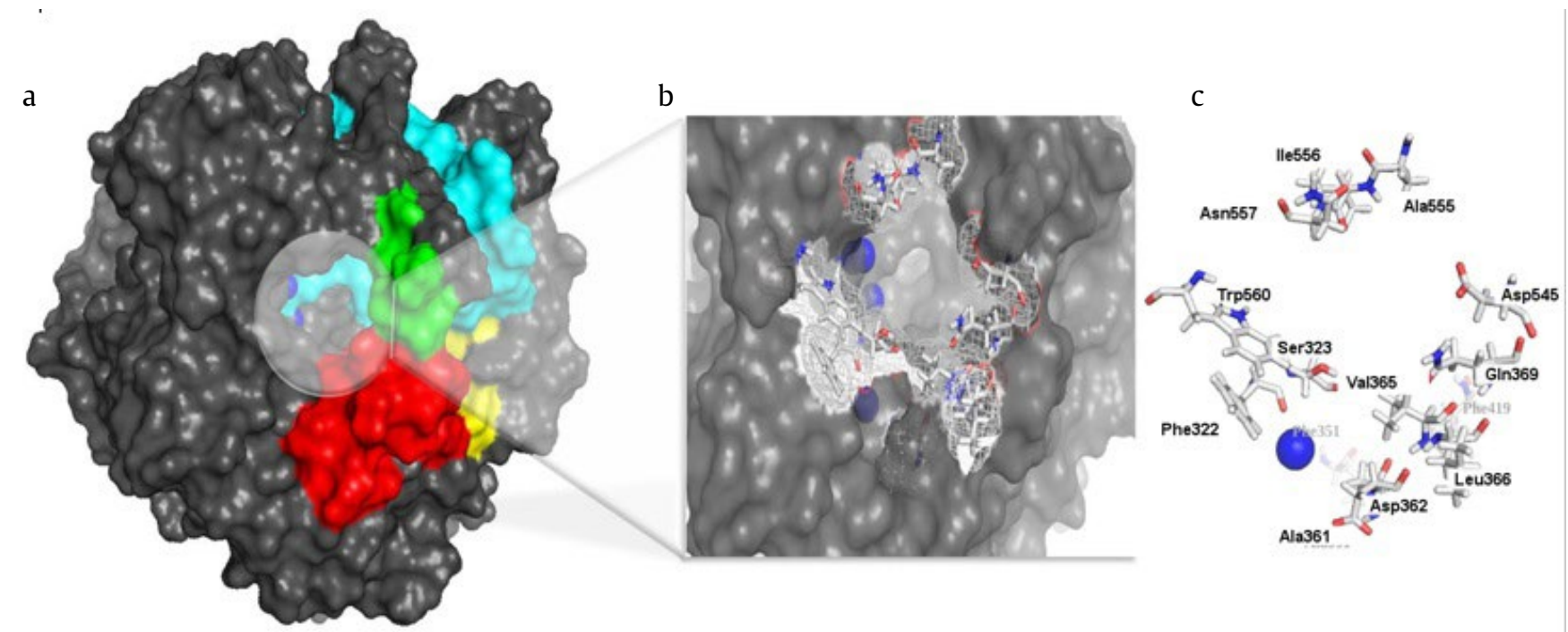

Figure 5. (a) The Substrate pocket and substrate binding loop of LAC inaCC. The Substrate-binding loop I is shown by red, loop II is shown by yellow, loop III is shown by green, and loop IV is shown by cyan, (b) the substrate pocket of the enzyme and their constituent amino acids, (c) the amino acid substrate pocket near the T1 copper site. The copper atoms are shown by a blue sphere, the amino acids are shown by stick form

Table 3. The structure quality parameter of the prediction structure of LAC inaCC before and after refinement by molecular dynamics simulation method (MD)

\begin{tabular}{|c|c|c|}
\hline Structure quality parameter & Before refinement \% & After refinement by MD \% \\
\hline \multicolumn{3}{|l|}{ Ramachandran Plot Statistics: } \\
\hline Most favored regions & 55.6 & 78.7 \\
\hline Additional allowed regions & 32.7 & 18.1 \\
\hline Generously allowed regions & 8.9 & 1.4 \\
\hline Disallowed regions & 2.8 & 1.8 \\
\hline \multicolumn{3}{|l|}{ Molprobity Summary Statistics: } \\
\hline Clashscore (all-atom contacts) & $33.47\left(12^{\text {th }} \text { Percentile }\right)^{\mathrm{a}}$ & 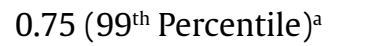 \\
\hline Molprobity score & $3.77\left(5^{\text {th }} \text { Percentile }\right)^{\mathrm{a}}$ & $1.70\left(89^{\text {th }} \text { Percentile }\right)^{\mathrm{a}}$ \\
\hline
\end{tabular}




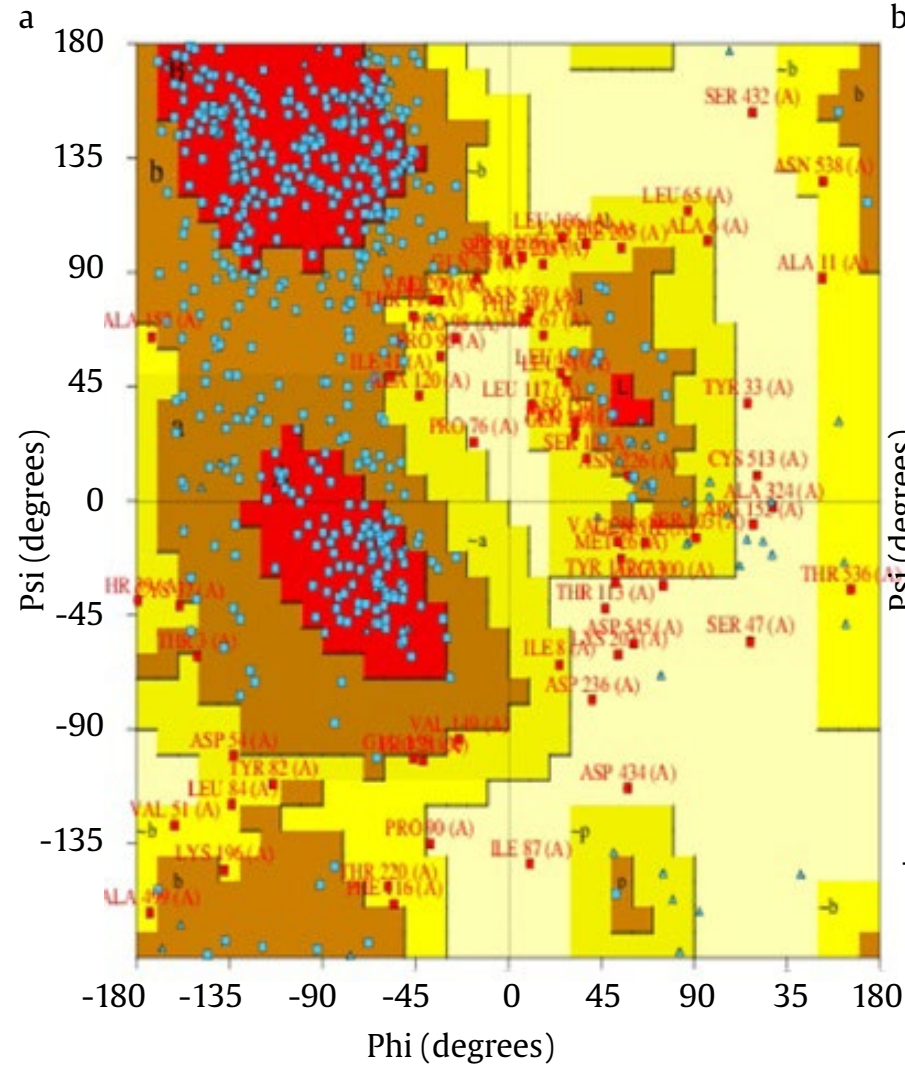

b

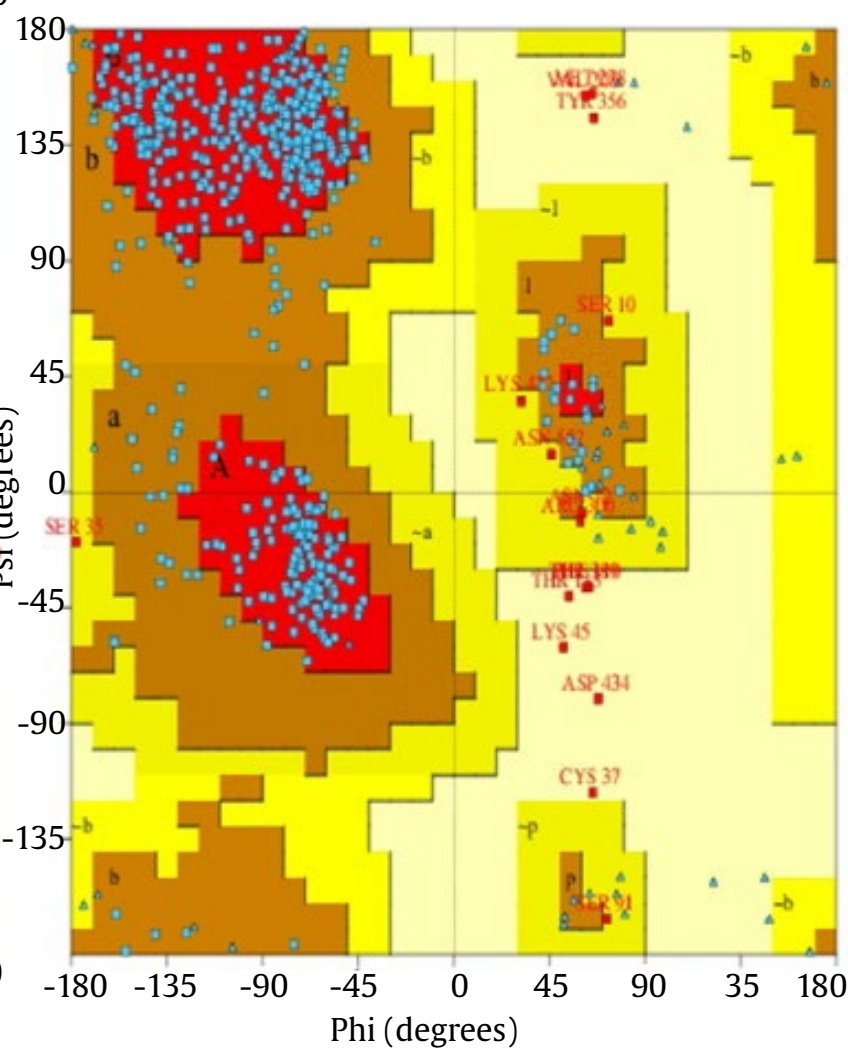

Figure 6. Distribution of torsion-angle of amino acids on the protein backbone of LAC inaCC by PROCHECK. a) The Ramachandran plot of the prediction structure of the enzyme before refinement by MD simulation method, $b$ ) the Ramachandran plot of the prediction structure of the enzyme after refinement by MD simulation method
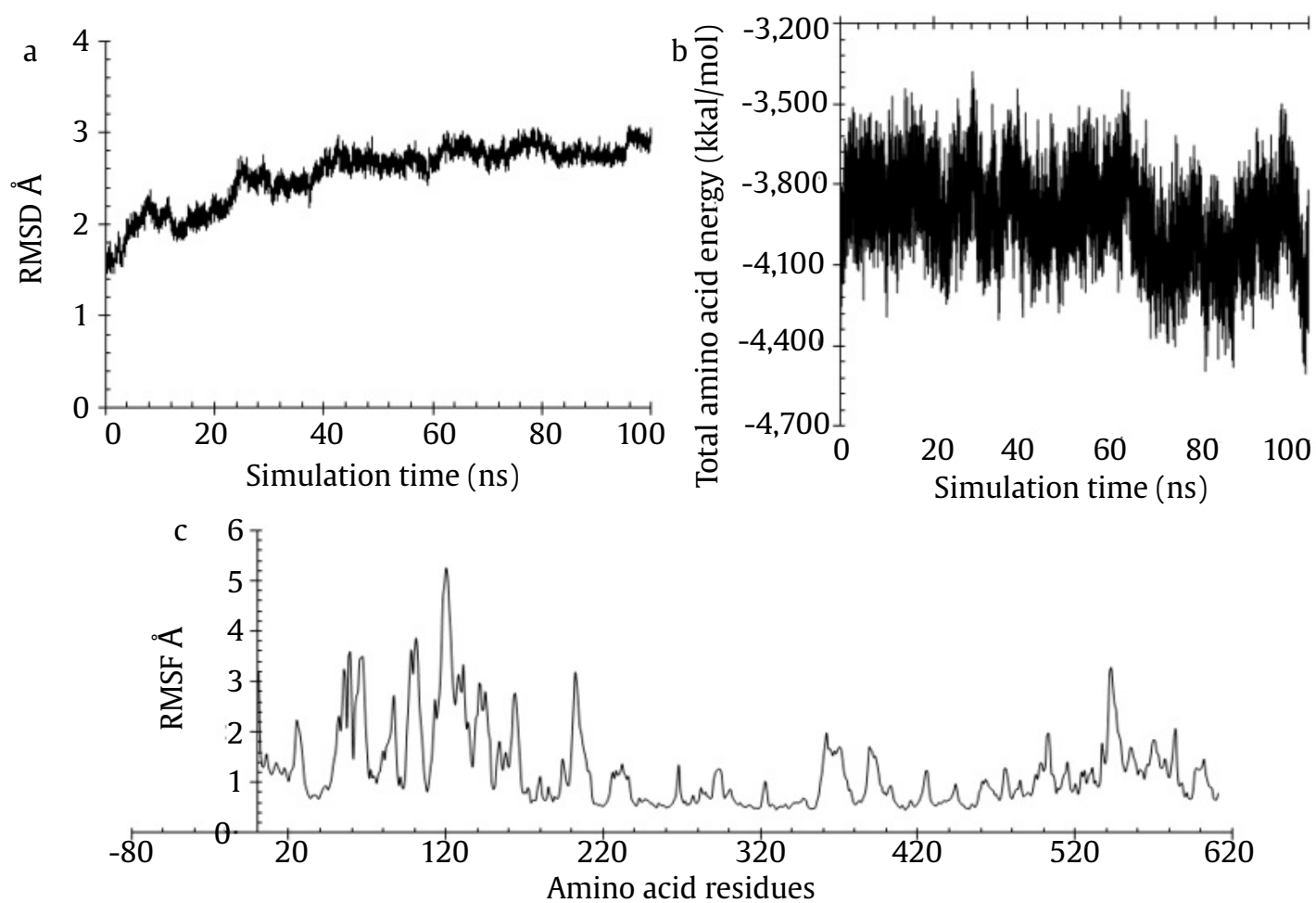

Figure 7. Molecular dynamic parameters of LAC inaCC for 100 ns time simulation. a) RMSD of C $\alpha$ atom, b) interaction energy of amino acids for 100 ns time simulation, c) RMSF of amino acids 


\section{Discussion}

Based on the homology result, the homology of the amino acid sequences of LAC InaCC was not high, only 63\% with Melanocorpus albomyces and Thielavia arenaria. Although the similarity percentage of its amino acid sequence belongs to the low homology category, the amino acid sequence of LAC inaCC can be concluded as homolog, based on sequence alignment in Figure 1. This was because the sequence alignment showed a number of sequences that were identical to the typical amino acid residue pattern called the sequence motif (as illustrated in Figure 1), in accordance with the provisions of Koonen and Galperin (2003). The sequence motif is an area consisting of a number of sequences that are conserved among proteins.

A conserved area means in each protein there is a certain amount of residue in the same typical pattern, which is a characteristic of a group of proteins (BenHur and Brutlag 2006). Sequence motif analysis is necessary to ensure the class of an enzyme (Ben-Hur and Brutlag 2006), especially LAC inaCC that has a low level of protein homology.

The first and the third sequence motifs ( $\mathrm{HWH}$ and HXXH) are known as the typical motif of laccases, commonly used to differentiate laccase from other enzymes in MCO superfamily (Kumar et al. 2003; Awatshi et al. 2014). Meanwhile, the second motif (HSH) is a typical motif of every enzyme in MCO superfamily. Based on the sequence alignment at Figure 1, tryptophan as a highly conserved residue was found between two histidines $(\mathrm{HWH}$ motif) in laccase sequence from Ascomycetes and Basidiomycetes fungi. Meanwhile, the phenylalanine residue was found in MCO enzyme from Ascomycetes fungi, Aspergillus niger. The tryptophan residue differentiates the laccase with other enzymes in the superfamily of multicopper oxidase. Those three sequence motifs found in LAC inaCC had the same sequence motifs with laccase from Ascomycetes fungi, Xylaria polymorpha, which plays a role as the binding site of copper atom (Liers et al. 2007). The existence of three sequence motifs with the typical pattern of $\mathrm{H}-\mathrm{X}-\mathrm{H}$ in the amino acid sequence of LAC inaCC proved that the isolated genes from Neurospora crassa inaCC F226 are genes encoding laccase based on typical characteristic of laccase sequences found (Kumar et al. 2003; Sirim et al. 2011; Garg et al. 2012; Awatshi et al. 2014).
On the sequence logo (Figure 2), the sequence motifs were seen. The conserved amino acid would have a high mark of bits in the sequence logo. Based on the results of Cooper-Binding Loop (CBL) analysis (Figure 3), proving that the sequence motifs has an important role in enzyme activity. All sequence motifs of LAC inaCC play a role in the binding of copper atoms (Awatshi et al. 2014). In the copper-binding loop, each copper atom was bond to histidine and serine which are sequence motifs of laccase through the hydrogen bond, forming the mononuclear site and the trinuclear site (Kumar et al. 2003; Giardina et al. 2009; Sirim et al. 2011; Awatshi et al. 2014), visualized in Figure 3.

On the other hand, LAC inaCC was proven to be an enzyme multisubunit consisting of three functional subunits known as cupredoxin-like domain (domain $\mathrm{A}, \mathrm{B}$, and $\mathrm{C}$ ), in Figure 4. Each domain plays specific roles in the catalytic process of laccase that are interconnected between domains (Trubitsina et al. 2015), thus the diminishing of one of the domains would cause laccase to lose its catalytic function. The site between domain A and B are used as a location for substrate oxidation of laccase involving one copper atom (T1 copper), called as the mononuclear site (Giardina et al. 2009; Jones and Solomon 2015). Meanwhile, the site between domains $\mathrm{A}$ and $\mathrm{C}$ provides function as a location to reduce the oxygen involving three copper atoms (T2/ T3 copper), called the trinuclear site (Giardina et al. 2009; Jones and Solomon 2015), visualized in Figure 4. The prediction structure of LAC inaCC showed typical structure motifs as laccase structure, it is the "Greek key $\beta$-barrel" structure motif at domains A and B (Hakulinen et al. 2002; Hakulinen et al. 2008; Giardina et al. 2009). Those characteristics proved that the prediction structure of LAC inaCC by using I-TASSER is the structure of laccase based on typical characteristics found.

The diversity of amino acid hydrophobicity in the substrate pocket (in Figure 5a and b) showed a wide substrate specificity (Ferraroni et al. 2012) in LAC inaCC. The wide substrate specificity caused the ability of laccase to oxidize various types of substrate, especially various polycyclic aromatic hydrocarbon (PAH) substances and also non-PAH ones, such as ABTS (Shraddha et al. 2011; Chrausia et al. 2013; Awatshi et al. 2014). The substrate-binding loop is closely related to the active site of the enzyme (Figure 5a). The substrate-binding loop plays a role in 
substrate binding and becomes the in-and-out track of the substrate-product. The substrate-binding loop determination referred to the research conducted by Giardina et al. (2009), stating that there were four substrate-binding loops in laccase.

Several important roles of the substrate-binding loop are to form some wall on the active site of laccase, to be expected as location for substrate binding during the catalysis process of laccase (Giardina et al. 2009), to open a space as location for interaction between substrate and copper atom on the mononuclear site, trinuclear site, and amino acid residues on the active site, the hydrophobic residue composing the substrate-binding loop in LAC inaCC has important structural function to maintain the protein conformation and hydrophobic nuclear stabilization (Pravda et al. 2014), the aromatic amino acids (Phe/F, Trp/W, Tyr/Y and Pro/P) and the charged amino acids (Asp/D, Glu/E, Lys/K, Arg/R, His/H) in the substrate-binding loop play roles as "gatekeeper" organizing the in-and-out track of substrate-product between the active site and surface of the enzyme through conformation alteration (Pravda et al. 2014; Pavlova et al. 2009).

The amino acid residue located on the axial position from the T1 copper (Figure 5c) atom plays an important role to determine the value of reduction-oxidation (redox) potential of laccase (Mate et al. 2010; Cambria et al. 2012). The existence of Phenylalanine (Phe) as laccase axial-ligand T1 copper, which can be seen in Figure $5 c$, would increase the redox potential value of laccase (Mate et al. 2010; Cambria et al. 2012; Pardo and Camarero 2015; Wang et al. 2015). Therefore, the LAC inaCC was expected to have higher redox potential value like laccases from other types of fungi (Cambria et al. 2012) with redox potential value of $700-800 \mathrm{mV}$ (Pardo and Camarero 2015). A high redox potential value would give an impact on the catalytic activity of LAC inaCC that can be developed in various fields of biotechnology (Fowler et al. 2017).

Based on Ramachandran plot result in the quality structure prediction assessment (Figure 6 ), the structure prediction of LAC inaCC had a low most favored region percentage of $55.6 \%$ with high disallowed regions percentage of $2.8 \%$. This showed that the structure conformation stability was low, the forming of inappropriate negative torsion-angle of phi- $\varphi$ and psi- $\psi$ in $C \alpha$ atoms of some amino acids. The appropriate torsion-angles to form the secondary structure are negative phi- $\varphi$ and psi- $\psi$ as much as $-60^{\circ}$ and $-50^{\circ}$ to form the helix, and torsionangle phi- $\varphi$ and psi- $\psi$ as much as $-140^{\circ}$ and $-130^{\circ}$ to form the beta-sheet (Hovmoller et al. 2002). The inaccuracy of torsion-angle in some amino acids caused overlap between atoms. High clashcore value (Table 3) indicated high atomic-overlap between amino acids (unfavorable all-atom steric overlaps) which is unfavorable to the quality of the protein structure. High molprobity value (Table 3 ) showed the resolution of LAC inaCC structure was low. The structure refinement process is required to improve the quality of this enzyme structure.

The increasing of most favored region percentage and the decreasing of sites outside the most favored regions percentage (the additional allowed regions, generously allowed regions and disallowed regions) in laccase structure after refinement through the MD simulation method. The value of the most favored region percentage increased significantly into $78.7 \%$ and the disallowed regions percentage decreased to $1.8 \%$ (Table 3 ).

It showed that several amino acids were experiencing the conformation alteration, which can be seen in Figure 6 . The clashcore and molprobity values also showed a significant increase, making each of them reached to $99^{\text {th }}$ percentile and $89^{\text {th }}$ percentile. It showed that the molecular dynamics simulation was able to improve the resolution and structure quality of LAC inaCC.

The increasing of RMSD value at the beginning of the simulation (Figure 7a) was supported by high fluctuation of positional alteration of some amino acid residues, in Figure $7 \mathrm{~b}$. It was caused by the conformation alteration from earlier structure which took place during the simulation to form several new secondary structures, such as forming four helical structures comprised of ${ }^{101} \mathrm{PSS},{ }^{123} \mathrm{VAGL},{ }^{153} \mathrm{QDSQ}$, and ${ }^{364}$ LVLLT helixes, and also five beta-sheets comprised of ${ }^{20} \mathrm{APQ},{ }^{27} \mathrm{QGQ},{ }^{199} \mathrm{RV},{ }^{204} \mathrm{SIL}$, and ${ }^{232} \mathrm{GVI}$ beta-sheets. The conformation alteration during the simulation was followed by fluctuation of interaction energy of the amino acid residues obtained from the electrostatic energy calculation, the Van der Waals energy, and the non-binding energy in Figure 7c. The highest SASA value as much as $4073 \AA 2$, based on the back-bone structure of protein. It showed that there was a significant conformation alteration in $\mathrm{C} \alpha$ atoms in each of amino acid residues during the simulation to form more stable conformation, causing the 
increasing value of most favoured region percentage on Ramachandran plot. The SASA value for nonpolar residue was $11839 \AA 2$, lower than the polar residues as much as 18073 A22. It showed a slightly strong interaction between non-polar amino acid residues, influencing the increasing of conformation stability of LAC inaCC, thus improving the quality of the structure.

\section{Conclusion}

The gene encoding LAC inaCC has been identified that the gene is the one coding the laccase enzyme based on the sequence motifs of $\mathrm{H}-\mathrm{X}-\mathrm{H}(\mathrm{HWH}, \mathrm{HSH}$, $\mathrm{HXXH}$ ) found. The sequence motifs play an important role in copper atom binding. The structure prediction of the enzyme has appropriate characteristics with laccase structure obtained through the experiment, although its level of similarity was low (65\%). This enzyme is a monomer of multisubunit enzyme, containing four copper atoms, and possessing the structural motifs of "Greek-key beta-barrel".

LAC inaCC expected to have broad substrate specificity due to its hydrophobic diversity in substrate-pocket, and high redox potential value. The molecular dynamics simulation was proven to be able to improve the structure quality of LAC inaCC, resulted from the I-TASSER prediction.

\section{Acknowledgements}

The authors acknowledge the Directorate General of Higher Education, Ministry of Research Technology and Higher Education, Republic of Indonesia for funding in the research program of Penelitian Dasar Unggulan Perguruan Tinggi (PDUPT), No. 1760/IT3.11/ PN/2018.

\section{References}

Awatshi M et al. 2014. Molecular docking and dynamics simulation analyses unraveling the differential enzymatic catalysis by plant and fungal laccase with respect to lignin biosynthesis and degradation. J Biomol Struct Dyn 33:1835-1849.

Ben-Hur A, Brutlag D 2006. Sequence motifs: highly predictive features of protein function. StudFuzz 207: 625-645.

Benkert P et al. 2011. Toward the estimation of the absolute quality of individual protein structure models. Bioinform J 27:343-350.

Cambria MT et al. 2012. In silico study of structural determinants modulating the redox potential of Rigidoporus lignosus and other fungal laccases. Biomol Struct Dynamics 30:89-101.
Chrausia PK et al. 2013. A review on mechanism of laccase action. Res Rev Biosci 7:66-71.

Dwivedi UN et al. 2011. Structure function relationship among bacterial, fungal, and plant laccase. J Mol Catal B:Enzym 68:117-128.

Feig M. 2016. Local protein structure refinement via molecular dynamics simulations with locPREFMD. J Chem Inf Model 56:1304-1312.

Feig M, Mirjalili V. 2016. Protein structure refinement via molecular-dynamics simulations: what works and what does not?. Prot 84:282-292.

Ferraroni M et al. 2012. Reaction intermediates and redox state changes in a blue laccase from Steccherinum ochraceum observed by crystallographic high/low X-ray dose experiments. J Inorg Biochem 111:203-209.

Fowler NJ et al. 2017. Prediction of reduction potentials of copper proteins with continuum electrostatics and density functional theory. Chem Eur J 23:1543615445.

Garg N et al. 2012. Cloning, sequence analysis, expression of Cyathus bulleri laccase in Pichia pastoris and characterization of recombinant laccase. BMC Biotchenol 12:1-12.

Giardina P et al. 2009. Laccase: a neverending story. Cell Mol Life Sci 67:369-385.

Hakulinen $\mathrm{N}$ et al. 2002. Crystal structure of a laccase from Melanocarpus albomyces with an intact trinuclear copper site. Nat Struct Biol 9:601-605.

Hakulinen N et al. 2008. A near atomic resolution structure of a Melanocarpus albomyces laccase. J Struct Biol 162:29-38.

Hovmoller S et al. 2002. Conformations of amino acids in proteins. Acta Cryst 58:768-776.

Jones SM, Solomon EI. 2015. Electron transfer and reaction mechanism of laccases. Cell Mol Life Sci 72:869-883.

Koonin EV, Galperin MY. 2003. Sequence-Evolution-Function: Computational Approaches in Comparative Genomics. Boston: National Institutes of Health.

Kumar SVS et al. 2003. Combined sequence and structure analysis of the fungal laccase family. Biotechnol Bioeng 83:386-384.

Liers C et al. 2007. Production, purification and partial enzymatic and molecular characterization of a laccase from the wood-rotting ascomycete Xylaria polymorpha. Enzyme Mircob Technol 41:785-793.

Mate D et al. 2010. Laboratory evolution of high-redox potential laccases. Chem Biol 17:1030-1041.

Pardo I, Camarero S. 2015. Laccase engineering by rational and evolutionary design. Cell Mol Life Sci 72:897-910.

Pavlova M et al. 2009. Redesigning dehalogenase access tunnels as a strategy for degrading an anthropogenic substrate. Nat Chem Biol 5:727-733.

Pravda L et al. 2014. Anatomy of enzyme channels. BMC Bioinformatics 15:1-8.

Shen $\mathrm{R}$ et al. 2015. Structural refinement of proteins by restrained molecular dynamics simulations with non-interacting molecular fragments.J PLOS Comput Biol 27:1-19.

Shraddha RSet al. 2011. Laccase: microbial sources, production, purification, and potential biotechnological applications. Enzyme Res 2011:1-11.

Sirim D et al. 2011. The laccase engineering database: a classification and analysis system for laccases and related multicopper oxidases. Database 2011:1-7.

Trubitsina LI et al. 2015. Structural and functional characterization of two-domain laccase from Streptomyces viridochromogenes. Biochimie 41:151159.

Wang J et al. 2015. Lignin engineering through laccase modification: a promising field for energy plant improvement. Biotechnol Biofuels 8:1-11. 